\title{
Optimizing the Number of Bluetooth Beacons with Proximity Approach at Decision Points for Intermodal Navigation of Blind Pedestrians
}

\author{
Jakub Berka, Jan Balata, Zdenek Mikovec \\ Faculty of Electrical Engineering, \\ Czech Technical University in Prague \\ Prague, Czech Republic \\ berkajak@fel.cvut.cz, balatjan@fel.cvut.cz,xmikovec@fel.cvut.cz
}

\begin{abstract}
Navigation in urban environments is very challenging for blind pedestrians. Although many navigation approaches using various principles or sensors to help visually impaired people exists nowadays they still have problems to navigate in complex buildings, find entrances to buildings or to navigate to correct public transport stops. Current solutions use a large number of sensor that needs to be installed in the environment needed to track every single move of the user. We present a solution to reduce the number of installed sensors by using previously developed set of landmark-enhanced navigation instructions allowing us to lower the necessary number of Bluetooth beacons by using them only for proximity notification at indoor decision points, indicating public transport station and entrances. The evaluation in the field study $(\mathrm{N}=8)$ suggests a good potential of the approach, especially in terms of usability, recovery from going astray and beacon deployment cost. Further, we provide guidance on beacons placement in the environment.

Index Terms-BLE, beacons, intermodal, navigation, blind pedestrians
\end{abstract}

\section{INTRODUCTION}

$\mathbf{F}$ OR the visually impaired people, it is vitally important to be able to travel independently and freely. The limitation in travel related activities has a negative impact on their quality of life and can result in worsening psychical condition and low self-esteem [1].

Many electronic devices, navigation aids, and navigation systems are now widely available for blind pedestrians. They are based on various principles of positioning such as Global Satellite Navigation System (e.g. GPS ${ }^{1}$, GLONASS $^{2}$ ) based systems for outdoor navigation (Blind Square, Ariadne GPS, Kapten Mobility); Bluetooth Low Energy beacons (NavCog), RFID $^{3}$ readers [2], or cameras [3] for indoor navigation. However, positional error for GPS in a city is about 28 meters for $95 \%$ of the time [4]. Similarly, indoor positioning systems often require high deployment costs and are not suitable to be used in other than indoor environments moreover, there is no global standard for indoor navigation systems yet.

In this paper, we propose a method for decreasing the number of Bluetooth beacons used for indoor positioning.

\footnotetext{
${ }^{1}$ Global Positioning System

${ }^{2}$ Global Navigation Satellite System

${ }^{3}$ Radio-frequency identification
}

We have identified various places on typical routes where the beacons are necessary because of complicated navigation and orientation, e.g. entrances to buildings, public transport stations, open spaces/areas (hallways, courtyards) and indoor decision points (location, where navigation instruction is needed because of multiple routes, can be taken such as corridor crossing). In the rest of the routes, we replace the beacons with landmark-enhanced navigation instructions, which provide necessary guidance when traveling. Instead of continuous location tracking, we use Bluetooth beacons for proximity estimation.

\section{RELATED WORK}

In this section, we describe approaches related to indoor and outdoor navigation of blind pedestrians. Furthermore, we focus on methods that use Bluetooth Low Energy beacons for navigation or proximity estimation.

\section{A. Pedestrian navigation of blind}

Successful navigation depends on the spatial knowledge about the environment. There are three levels of environment knowledge applied for navigation in cities: knowledge of landmarks, knowledge of route and overview knowledge [5]. Landmarks can be defined in various ways as says Golledge in [6], a landmark is something capable of attracting attention, i.e. it has dominance visible form and stands out from the surrounding environment. For navigating visually impaired people outdoors the suitable landmarks can be e.g. street corners and their different shape, pedestrian crossings, steps, etc. For indoor navigation visually impaired people still use the landmarks because the three levels of environment knowledge are valid indoors as well, only the characteristic of landmarks change (e.g. door, stairs, type of rooms).

\section{B. Bluetooth Localization}

Work of Gorovyi et al. [7] shows the application of beacons for real-time users positioning based on trilateration calculation using Received Signal Strength Indicator (RSSI) values from three or more beacons. Performed accuracy test showed 
that beacon calibration improves system efficiency (1-2 meters in their case).

The Bluetooth technology used for indoor localization is often combined with utilization of other sensors to improve the accuracy of the navigation in an indoor environment (Accelerometer, Barometric sensor). Czogalla and Naumann [8] developed indoor navigation for $8000 \mathrm{~m}^{2}$ public indoor environment with 35 beacons installed. The route is presented to users by visual map and directions by vocal instructions.

Commercial solutions for indoor location or positioning based on the beacons often use a triangulation/trilateration approach such as Indoo.rs ${ }^{4}$. The difficulty of these solutions is mainly in a large number of beacons required. To achieve their proposed accuracy 1-3 meters it is necessary to install beacon every 7-10 meters, e.g., with Infsoft indoor navigation ${ }^{5}$.

\section{Indoor Navigation for Blind}

Work of Ahmetovic et al. [9] resulted in NavCog system, which relies on Bluetooth beacons installed in an environment and provides sub-meter precise localization with a minimum of 1 beacon every 6 meters and navigation assistance for people with visual impairments. They achieve it by representing the environment in the one-dimensional graph which results in lower number of beacons to be used and further, they use multi-modal probabilistic state estimation algorithm and Particle Filtering framework to more precisely estimate user's position. NavCog system gives to user the "turn-by-turn" metric navigation instructions, distance announcements inform the user about the distance to next action (e.g. "18 meters"), action instructions give information about turning direction or transit information (e.g. move between floors). It also provides accessibility instruction (e.g. if there is a curb that is easy to trail with a cane) or surrounding information (e.g. building description) on request.

To help visually impaired children in school to move and play independently Freeman et al. [10] used Audible Beacons as wearable bracelets that support wireless communication and provide audio output. Beacons are also placed in the school environment. They presented various scenarios based on their solution. Beacon bracelets can inform children about their nearby points of interest, by playing a specific sound of this $\mathrm{POI}^{6}$. As the children get closer to the POI the sound is played louder. It can help to find sighted friends, who are wearing bracelets as well. Bracelets and beacons placed in the environment can help to learn the layout of the school including entrances.

Guo et al. [11] developed Landmark-based Mapless Indoor Navigation called FreeNavi that requires only WiFi fingerprints collected on the device. This system applies knowledge of humans being able to navigate through and identify the environment by landmarks. FreeNavi constructs a virtual map only by landmarks descriptions and their connectivity relations. Virtual map construction algorithm is based on $\mathrm{WiFi}$

\footnotetext{
${ }^{4}$ Indoo.rs - https://indoo.rs/

${ }^{5}$ Infsoft - https://www.infsoft.com/

${ }^{6}$ Point of Interest
}

signal strength data and also landmark fingerprints and the user traces, this data is crowdsourced and then the map created. The generated map does not contain information of turning directions (left or right), i.e. users have to find out by themselves at the junctions in which direction they have to continue to next landmark.

Finding entrances to the desired building was subject of researchers focused on crowdsourcing. The study says that almost $65 \%$ of blind and visually impaired people suffered the mobility hindrance of hard to find entrances in the international survey by Zeng and Weber [12]. To solve this issue authors used collaborative method for collecting information about entrances and buildings and they also created reference point for each entrance, their concept does not use GPS data but it is expected to use some GPS-base tool to navigate to reference point, when user approaches the entrance s/he gets structured and also unstructured collected information by other users. [12].

Our proposed solution is based on automatically generated landmark-enhanced navigation instructions for outdoor [13], indoor [14] environments and their combination for different environments transitions and usage of public transportation [15]. For automatic generation of the navigation instructions it is necessary to use specially modified GIS which contains information about sidewalk network and special features of the urban environment (slope, surface quality, railings, corners) as it is presented in [13]. In our work, we aim to address the issue of finding the entrances [12], as well as a large number of installed Bluetooth beacons [9] by using them only for notification of progress at decision points without trilateration.

\section{NAVigation Application Prototype}

We developed a prototype of the navigation application which provides navigation instructions for outdoor, public transport and indoor navigation for blind pedestrians. The transition between different modes are seamless - at the transition from one mode/environment to the other, the application provides summarized description of the mode/environment such as the description of the public transport lines and stations or description of the building. In selected locations, the application provides notification about user's progress on the route triggered by Bluetooth beacons.

\section{A. Route itinerary}

The route itinerary contains detailed navigation instructions for each segment of the route, which consists of a description of the surrounding environment and action to be performed by the user. Construction of navigation instructions is based on the sidewalk-based GIS $^{7}$ for outdoor environments [13], template system for public transport stops and stations and environment transitions (e.g., from outdoor to indoor) [15] and landmarkenhanced navigation instructions for indoor [14]. Navigation instructions are stored in the navigation application in the graph data structure (see Fig. 1 and Tables I, II).

\footnotetext{
${ }^{7}$ Geographic Information System
} 


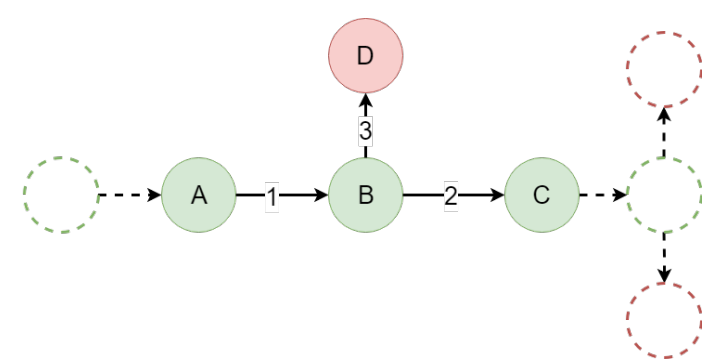

Fig. 1. Example of the generated graph structure. The detailed information about the content of the nodes and edges are presented in Tables I, II.

TABLE I

EXAMPLE OF THE NODES DATA IN GRAPH DATA STRUCTURE.

\begin{tabular}{|c|c|c|}
\hline Node & isOnRoute & BeaconID \\
\hline $\mathrm{A}$ & true & undefined \\
\hline $\mathrm{B}$ & true & ODUM \\
\hline $\mathrm{C}$ & true & vf3i \\
\hline $\mathrm{D}$ & false & $4 \mathrm{vXL}$ \\
\hline
\end{tabular}

\section{B. Beacons Installation}

The navigation application uses RSSI from beacons to estimate its proximity. There are several factors influencing the signal transmission: the signal can be absorbed by other persons moving nearby beacons; the signal can be interfered by other devices working on the same frequency spectrum as beacons $(2.4 \mathrm{GHz}$, same spectrum used by Wi-Fi 802.11 ); many materials can act as barriers for Bluetooth signal such as plaster, concrete, bulletproof glass, or metal materials ${ }^{8}$. These limitations have to be taken into account during beacon installation.

In the indoor environment, the beacons are installed only at the decision points - corridor junctions, corridor bents, floor mezzanines and complicated stair system. We placed the beacons mainly on the walls $2.5-3.5$ meters above ground (see Fig. 2).

At public transport stations, the suggested place for installing the beacon is the info-table. Same as in indoor environment the beacon should be placed above the people's heads. In our case, we placed it at the tram station oriented towards the sidewalk.

To help users with environment transitions we placed the beacon near the building entrances. The beacon should be placed with respect to possible directions of the user's approaches to the entrance. If possible, as much as possible in the sidewalk level. When placing the beacon at the entrance our intention was first to slow down or stop the user. Second to let the user read the detailed description of the entrance from route instructions and find the correct door.

Moreover, we placed the beacon at the decision point in the semi-outdoor environment (university campus courtyard). This environment is composed of the roadways rather than sidewalks, and the users have to navigate through open spaces.

\footnotetext{
${ }^{8}$ Apple - https://support.apple.com/en-us/HT201542
}

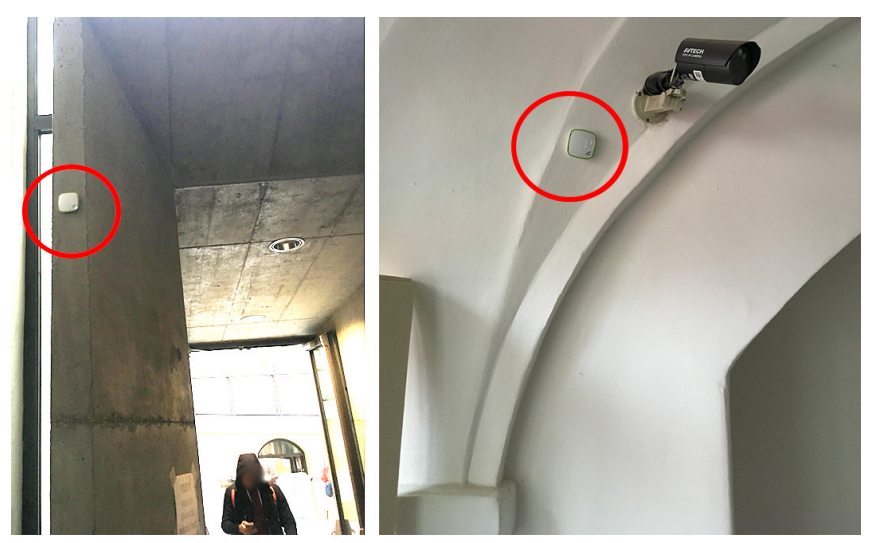

Fig. 2. Beacons installation at the suitable places.

To help them find the decision point at the roadway turns, we placed the beacon on the building near it at 3,5 - $4 \mathrm{~m}$ high.

After the beacons installation, we configured beacons to avoid interferences of two or more beacons in mind and adjusted advertising interval and transmission power to ensure sufficient signal coverage. Finally, we collected RSSI to determine the thresholds to trigger location about the progress on the route.

\section{Route Progress Notifications}

The route is represented as an oriented graph.

The graph has two types of the nodes, correct and error. The correct nodes represent the decision points on the generated route. The error nodes currently used only for indoor segments represent the wrong turns off the route. E.g. if the route contains junction of corridors with one correct turn-off and two wrong turn-offs the graph will have one correct node and two error nodes. If there is a beacon installed at a particular decision point, its ID is stored in the node. For outdoor segments of the route, we store IDs only for nodes at entrances and public transport stops.

The edge represents a route segment. Edges on the correct route hold data about the segment number and the navigation instruction. Edges off the correct route lead to an error node. The part of the route graph we created for the evaluation purposes can be seen in Fig. 3 .

We use the proximity-based approach - beacons serve as proximity traps at decision points, therefore the number of installed beacons is highly reduced. Route graph representation enables us to provide users with error prevention and also error recovery at the more complicated decision points - when the user goes astray s/he is notified and can use backtracking to get back on the route. Beacons ID are tied to navigation instruction displayed on a screen of the phone.

\section{User Interaction}

The navigation application was implemented in multiplatform Ionic framework version 3. The user interface provides controls for navigating between individual navigation instruction and for manual location verification. The user can 
TABLE II

EXAMPLE OF THE EDGES DATA IN GENERATED GRAPH DATA STRUCTURE.

\begin{tabular}{|c|c|c|c|c|}
\hline Edge & is OnRoute & Segment Nr. & Segment Description & Segment Action \\
\hline 1 & true & 1 & $\begin{array}{c}\text { You are at the turning of the road, } \\
\text { the building E is in front of you. }\end{array}$ & $\begin{array}{c}\text { Turn left and go approximately 30 meters } \\
\text { through open space to pyramidal stairs, } \\
\text { by your right hand. } \\
\text { an entrance to the building E. }\end{array}$ \\
\hline 2 & true & 2 & $\begin{array}{c}\text { You are by the entrance to the building E. } \\
\text { The pyramidal stairs there is big wooden door } \\
\text { and glass swing door right behind, } \\
\text { leading inside the building. }\end{array}$ & $\begin{array}{c}\text { Go up the stairs and through the doors } \\
\text { inside the building. }\end{array}$ \\
\hline 3 & false & undefined & undefined & $\begin{array}{c}\text { You are on the wrong way, } \\
\text { return back to the beginning of the segment. }\end{array}$ \\
\hline
\end{tabular}

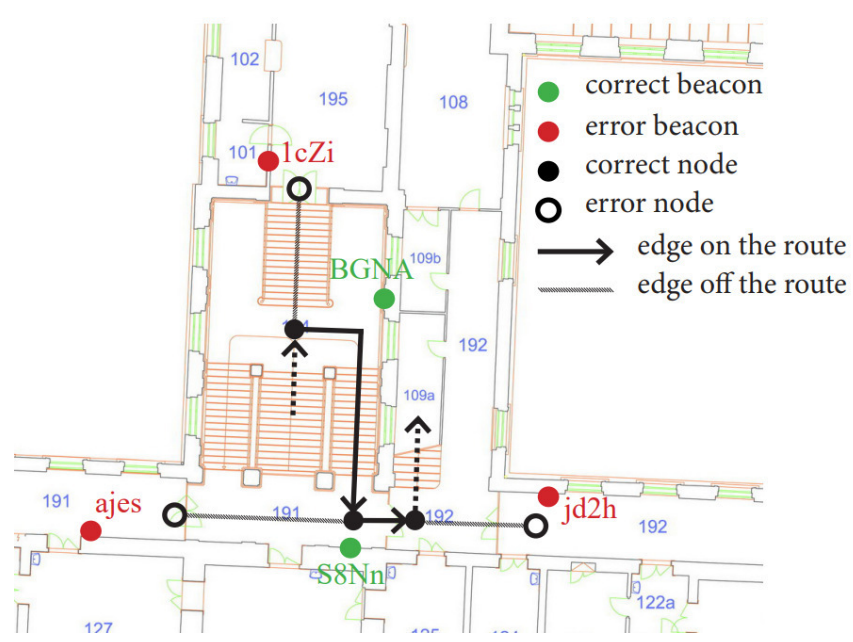

Fig. 3. Part of the experimental route represented as an oriented graph. With positions of installed beacons.

navigate between individual navigation instruction by pressing the buttons "Next Segment" and "Previous Segment".

When the user approaches the correct beacon, the application automatically notifies the user by vibration and text "You are near the end of the segment." Similarly, when the user turns off the route and approaches in the nearby of the error beacon from an actual segment, the application will four times short vibrate and announce to user "You are on the wrong way, return to the start of this segment."

These two notifications from application happen automatically when the user is in the vicinity of the corresponding beacon. However, the application also provides the possibility to manually verify user's position. When the user presses "Verify location" button the 5 seconds timeout starts and application is ranging for correct or error beacons. When the 5 seconds limit expires and the user is not in the nearby of any beacon application will announce "Verification of your location was not successful."

\section{Evaluation}

The navigation application supported one experimental route with an outdoor-indoor combination and usage of public transportation. Navigation instructions were automatically generated for outdoor (using Naviterier ${ }^{9}$ ) and manually prepared for the indoor, public transport and environment transitions.

\section{A. Participants}

Eight visually impaired participants were recruited via email leaflet (in the leaflet we mentioned that touchscreen smartphone will be used for the experiment). They were aged from 33 to 53 years ( $m e a n=40.25, S D=7.27$ ). Five participants were congenitally blind and 3 were late blind. Three participants had Category 4 visual impairment (light perception) and 5 participants had Category 5 (no light perception) [16]. One participant had a guiding dog. All of the participants were native Czech speakers.

\section{B. Apparatus}

Route. The experimental route was located in city centre of Prague, Czech Republic with the combination of outdoor, semi-outdoor and indoor environments and use of public transportation (ride with tram).

It was approximately $700 \mathrm{~m}$ long (excluding tram ride), consisted of 36 segments. Twenty six Beacons Pro and 1 Tough Beacon ${ }^{10}$ were installed on the route in total, 16 beacons were placed on the route decision points and 11 beacons were placed on the turnoffs from the route. The beacons for public transport station and at the first entrance were held by $2^{\text {nd }}$ experimenter glued on the paper folder above the head (as we did not get permission for long term placement).

Equipment. The participants were equipped with HTC One 801n smart-phone - Android 5.0.2, with running Talk Back screen reader set to Czech language and with the installed navigation application. The smart-phone had a lanyard that participant could hang on his/her neck, to have free hands when necessary. We also gave participants chip card that is needed to open a door inside the university campus buildings.

Data Collection. During each route walk-through, we were shadowing participants and recording third-person video. The navigation application records a log file containing data with timestamps about the interaction with the application, i.e. button presses, location notifications (triggered automatically)

\footnotetext{
${ }^{9}$ Naviterier - https://www.naviterier.cz/en

${ }^{10}$ Kontakt.io - https://kontakt.io/
} 


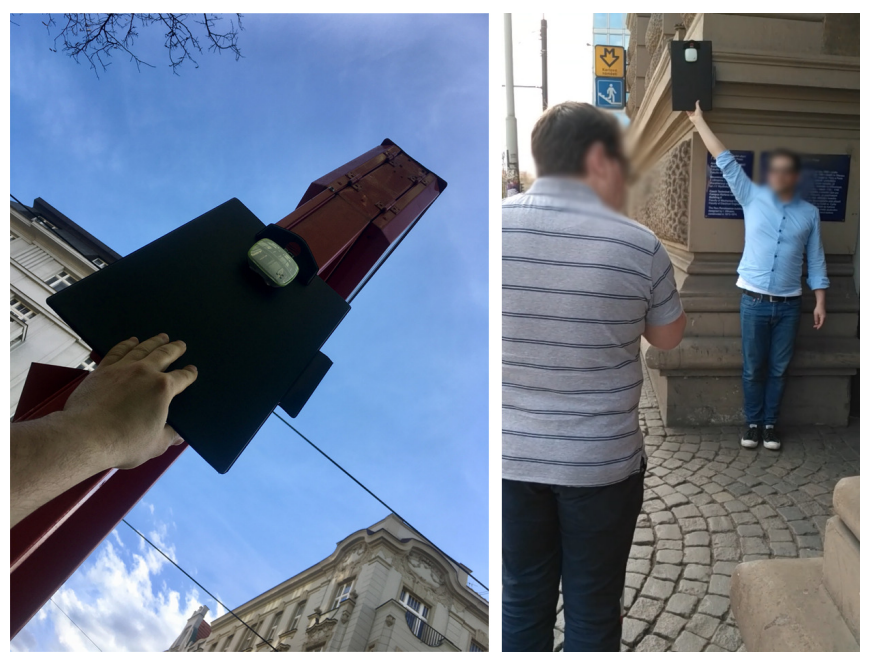

Fig. 4. Photos from tram station and entrance to first building, show how the second experimenter hold the beacon.

and location verification requests (triggered by the user), and data about ranged beacons during the walk-through, with information about beacon ID and current RSSI.

\section{Procedure}

The experiment lasted around 1 hour. At first, we explained to the participant the purpose of the experiment, collected the demographic information and explained the operation and control of the application.

Then we accompanied the participant to the start of the route and we explained the task: "Imagine, you were invited to participate in a study at the University Campus in a building E, room 317. To reach the destination use the navigation application, which will give you navigation instructions and it will notify you whether you are going the correct way at public transport stops and at indoor locations where you will also be notified if you go astray.". They were instructed to proceed as if they were alone. However, whenever they felt unsafe, they should ask for our assistance.

After the participant finished the route, s/he was debriefed and asked about subjective judgement about the level of safety ("I felt safe during the route walkthrough."), efficiency ("I think that thanks to application and navigation instructions I proceeded efficiently."), information sufficiency ("The Information I received from application was sufficient."), comprehension ("The instructions in decision points of the route were comprehensive.") and confidence when finding entrances ("I felt confident thanks to application when finding entrances to buildings.") and tram station ("I felt confident thanks to application when finding tram station.") on a 5 point Likert scale as a level of agreeing with presented statements.

\section{Results}

All participants successfully completed the route. The average completion time was 44.2 minutes ( $\mathrm{SD}=8.7$ minutes).

\section{A. Tram station}

Participants P1, P6, P8 found the tram station successfully, the beacon triggered the automatic notification.

Further, participants P2 and P3 misheard the notification from the application when finding the tram station, but the manual verification of location afterward was successful and helped then find the tram station. P7 also misheard the notification from the application, missed the info table at tram station, and continued further on the sidewalk (went astray) until we stopped him and returned him to the route.

Participants P5 and P8 confused the telephone booth, which was about 20 meters before the tram station with the info table of the tram station. They both tried to verify the location there and the application correctly responded. Afterward, P5 was able to find the tram station without the location verification as he switched to next segment early and P8 found it successfully with help from automatic notification from the navigation application.

Participant P6 was impressed by the automatic notification at the tram station, he said: "As a blind when I am finding the tram station I have to walk near the building due to public notice so it is hard to find the station at the sidewalk, the automatic notification is very helpful." P8 had the same opinion about stations: "It is not necessary to receive the notifications everywhere but at tram station or near the entrances it is very good."

\section{B. Semi-outdoor.}

At the courtyard, P1 did not follow the curb curved to the right and he continued in a straight direction. He complained about the missing information about curved curb. P3 and P4 skipped to the next segment before they reached the turn, but they continued without problems. Participants P6 and P7 had trouble with the manual location verification as they tried it few meters after they passed the beacon. P7 did not receive the automatic notification when he was near the beacon on the road turn however he continued without problems. Rest of the participants did not have problems when walking through the university courtyard and received the location notification.

After the experiment P7 explained that he lacked information about the distance and direction to the beacon: "I would welcome the information about the distance to the decision point, now I am not sure if it is in front or behind me."

\section{Entrances}

Entrance to building A (in the second niche of the protruding facade): Participants P1, P3, P4, P5 and P6 found the correct entrance to the first building. P3 found the entrance without the automatic notification but afterward verified the location manually twice and twice it was successful. P2 missed the entrance to the first building, the beacon triggered late, after a while we stopped him and returned him back to the route. P7 and P8 missed the entrance to the first building, the beacon did not trigger. P7 tried to verify location manually in the first niche, but the beacon did not trigger. 


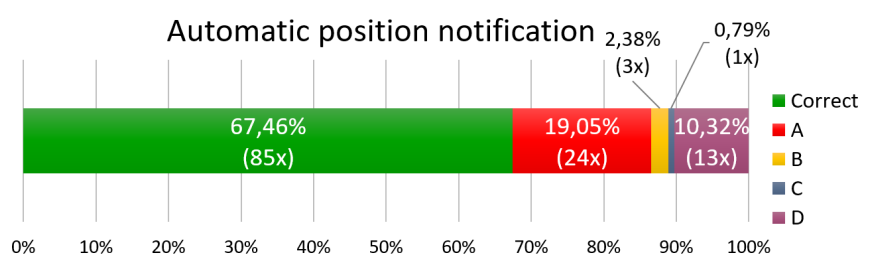

Fig. 5. Results of automatic notifications at decision points (total=126).

Entrance to building E (with pyramidal stairs): Participants P1, P2, P5 and P7 found the entrance with help of automatic notification from the application. P3 found the entrance but skipped to the next segment too early before the beacon can trigger. $\mathrm{P} 4$ found the entrance but the beacon did not trigger. P6 was proceeding to the entrance along the building and so the signal from beacon was shielded by the building, but participant found the entrance correctly. P8 stopped near the entrance when he got the notification at the right moment, but then continued straight and missed the stairs on the right, because he was proceeding on the very left side of the road during the segment leading to entrance stairs.

\section{Indoor}

Only P2 (once) and P4 (twice) went astray during the walkthrough indoors. The error beacons triggered correctly and participants were notified that they should return to the start of the segment. P2 recovered from the error successfully alone. P4 at first recovered with an assistance and on the second error turn recovered alone successfully. P1, P3, P5, and P8 needed an assistance when finding the chip card reader next to the door. All participants but P5 found the correct door in the final segment.

Participant P6 mentioned that "The vibrations and notifications were very accurate, it vibrated where it should for example at the last step of the stairs." P3 noted that it would be beneficial at public transport stations and building entrances and in large buildings "It would be great to have it for example in big hospital compounds." Participant P2 was not satisfied with automatic notification at several indoor parts of the route: “... in atrium it gave me the notification too early."

\section{E. Beacons}

The automatic notification about the user position near the beacon was expected to happen 126 times in total during the whole experiment with 8 participants. Not always was the notification triggered as expected. In Figure 5 we present the results of the automatic triggering. The reasons why the automatic notification failed are various, e.g. signal interference, bad configuration of a beacon. Bellow, we present the detailed list of the different types of failures.

A: Notification did not trigger, near the beacon. Due to signal distortion or interference, bad configuration of the beacon or the signal covered by participant's body when turned away from the beacon. In two cases the users were behind the thick wall which absorbed the beacon signal.

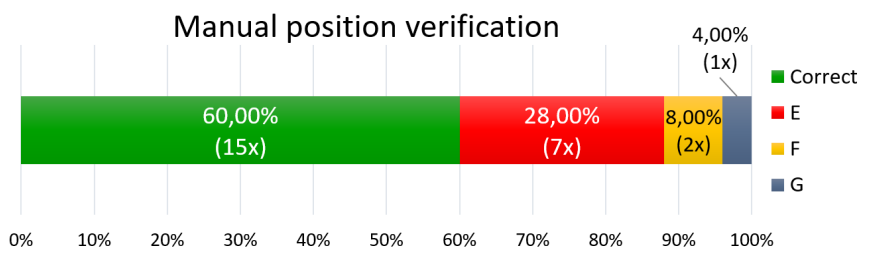

Fig. 6. Results of the manual position verification (total=25).

B: Notification triggered too early, on the beginning of the actual segment. It happened during the short segments indoors. The distance it should trigger was to approximately 4 meters, but it triggered 8-10 meters away. The signal could be mirrored by a metal surface.

C: Notification triggered too late, when the participants just passed the end of the segment, therefore, they missed the decision point.

D: The application is ranging only for beacons in particular segment displayed on the screen. For more information see Section III-D. Some of the participants were reading the segments in advance and did not return to the actual segment in the application. We do not evaluate this situation as a failure.

The manual verification was used by participants 25 times in total during the experiment. Some participants tried to verify their location after they received the automatic notification and continued few steps ahead. The manual verification then could fail, because they stepped out of the beacon's range, or they covered the signal with their body. In Figure 6 we present the results of the manual position verification. Bellow is the list of various types of the manual verification failures.

E: Manual verification did not trigger the notification, near the beacon. Due to the signal distortion or interference, bad configuration of the beacon or the signal covered by participant's body when turned away from the beacon.

F: Manual verification failed, after the correct automatic notification. Participants stepped out of the range of the beacon, or the signal was covered by their own body.

G: Manual verification was successful on the second attempt.

\section{F. Subjective judgement}

Fig. 7 shows that $50 \%$ of the participants strongly agreed on information sufficiency they obtain from the application, one participant disagreed. $63 \%$ of the participants strongly agreed on comprehension at the synchronization points, one participant disagreed. $38 \%$ of the participants agreed about confidence when finding entrances, 2 participants disagreed. $63 \%$ of the participants agreed about the confidence when finding the tram station, 2 participants disagreed. $25 \%$ strongly agreed on safety and efficiency, one participant disagreed on safety and one disagreed on efficiency.

\section{G. Discussion}

The results clearly show that correct setting of the beacons is crucial for the proper functioning of this navigation system. 


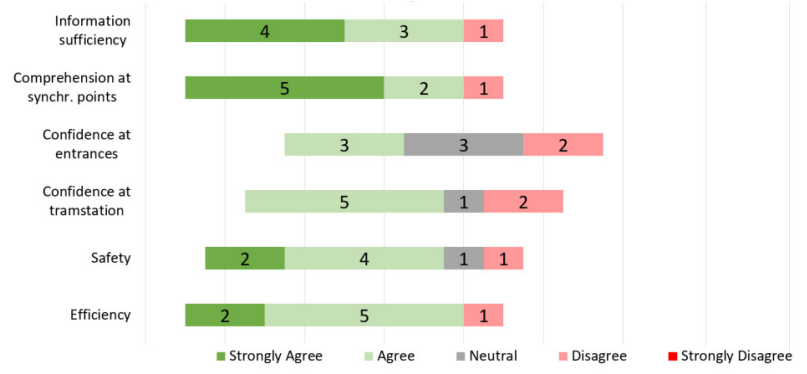

Fig. 7. Subjective judgements about level of information sufficiency, comprehension, confidence, safety and efficiency $(\mathrm{N}=8)$.

When placing a beacon at the entrance to a building, it should be remembered that users can come from different directions and that all of them should be covered by the signal. If it is a proportionally complicated entrance, it can be considerate to place a larger number of beacons to cover user's arrivals from all directions. It is not necessary to have beacons installed on each segment, sometimes it is not even physically possible, specifically on very short segments there may be a problem that the correct and error beacon will be very close to each other, which may result in an error location notification to the user even he is near the correct beacon or vice versa.

We experienced that there can be spaces where it is really difficult to correctly configure the beacons. In our case, it was the atrium with metal glass construction connecting two buildings. We tried to place there two beacons on the opposite sides of the atrium. But the signal distortion was too high, we decided to install there only one beacon to prevent the signal interference with the second beacon.

During the evaluation, some of the beacons triggered the position notification too early or too late. This issue can be solved either by the reconfiguration of the beacon or by moving the beacon 1 meter forward/backward.

The automatic notification often did not trigger because the user switched to the next segment. In our current solution the application is ranging the beacons only for the currently displayed segment. In future, this should be improved and the user notified about the surrounding information when arrives near any of the beacons.

Two of the participants took the wrong turn during the evaluation. They were able to recover from this error. We can say that even without the distance and direction information our proposed combination of navigation instructions with beacons only at decision points can solve the user's walkthrough errors using backtracking.

The manual location verification feature did not result in higher reliability. The participants tend to stop using this feature during the walkthrough. It happened that the automatic verification of the location worked correctly, but the manual verification afterward did not. The main reason was that the participant crossed the decision point by few steps and tried to verify the location beyond the beacon range. This could have influenced the user's confidence level at the decision point. We think that this feature can be omitted from the application.

If we compare our approach to NavCog [9] in terms of the number of beacons necessary, for the indoor and semi-indoor (courtyard) parts of the route (250 meters and 26 segments) we needed 26 beacons in total including error beacons. If we placed the beacons every 6 meters we would need approximately 42 beacons to cover only the route excluding error beacons.

\section{CONCLUSION}

We designed a prototype of navigation application that has two main building blocks, the landmark-enhanced navigation instructions and the location synchronization system that uses the minimum number of beacons possible and that is also capable of error prevention and error recovery.

We conducted a qualitative study of high-fidelity prototype of this system with 8 visually impaired participants. As previous studies shown [13] the landmark-enhanced navigation instructions are suitable navigation for blind pedestrians, still there are many difficulties which we wanted to solve with utilizing beacons as synchronization points, i.e. finding entrances, public transport stations, help when identifying the landmarks and give users more confidence during the walk-through.

As we found out, the synchronization points successfully complement the navigation system using only navigation instructions. The main benefit of our solution lies in the use of a low number of beacons. But maintaining the effectiveness of navigation thanks to detailed and landmark-enhanced navigation instructions.

For the future, we see a potential in installing the beacons only on the most used and hard-to-find decision points namely at public transport station and entrances to public buildings.

\section{ACKNOWLEDGMENT}

The research has been supported by projects: Navigation of handicapped people funded by grant no. SGS16/236/OHK3/3T/13; Research Center for Informatics (OP VVV CZ.02.1.01/0.0/0.0/16_019/0000765); Automated mapping of routes and barriers for pedestrians and disabled people funded by grant no. TH02010839 of the Technology Agency of the Czech Republic realized by Central European Data Agency, a.s.

\section{REFERENCES}

[1] R. G. Golledge, "Geography and the disabled: a survey with special reference to vision impaired and blind populations," Tran. of the Inst. of British Geographers, pp. 63-85, 1993. [Online]. Available: https://www.jstor.org/stable/623069

[2] J. Faria, S. Lopes, H. Fernandes, P. Martins, and J. Barroso, "Electronic white cane for blind people navigation assistance," in WAC 2010. IEEE, 2010, pp. 1-7.

[3] M. Bujacz, P. Baranski, M. Moranski, P. Strumillo, and A. Materka, "Remote guidance for the blind - A proposed teleassistance system and navigation trials," in HSI 2008. IEEE, 2008, pp. 888-892. [Online]. Available: https://doi.org/10.1109/HSI.2008.4581561

[4] M. Modsching, R. Kramer, and K. ten Hagen, "Field trial on GPS Accuracy in a medium size city: The influence of built-up," in 3rd workshop on positioning, navigation and communication, 2006, pp. 209218 . 
[5] A. W. Siegel and S. H. White, "The development of spatial representations of large-scale environments." Adv. in child development and behavior, vol. 10, p. 9, 1975. [Online]. Available: https://doi.org/10.1016/S0065-2407(08)60007-5

[6] R. G. Golledge, "Human wayfinding and cognitive maps," Wayfinding behavior: Cognitive mapping and other spatial processes, pp. 5-45, 1999.

[7] I. Gorovyi, A. Roenko, A. Pitertsev, I. Chervonyak, and V. Vovk, "Realtime system for indoor user localization and navigation using bluetooth beacons," in 2017 IEEE First Ukraine Conference on Electrical and Computer Engineering (UKRCON), May 2017, pp. 1025-1030. [Online]. Available: https://doi.org/10.1109/UKRCON.2017.8100406

[8] O. Czogalla and S. Naumann, "Pedestrian indoor navigation for complex public facilities," in 2016 International Conference on Indoo Positioning and Indoor Navigation (IPIN), Oct 2016, pp. 1-8. [Online]. Available: https://doi.org/10.1109/IPIN.2016.7743672

[9] D. Ahmetovic, M. Murata, C. Gleason, E. Brady, H. Takagi, K. Kitani, and C. Asakawa, "Achieving Practical and Accurate Indoor Navigation for People with Visual Impairments," in Proceedings of the 14th Web for All Conference on The Future of Accessible Work, ser. W4A '17. New York, NY, USA: ACM, 2017, pp. 31:1-31:10. [Online]. Available: http://doi.acm.org/10.1145/3058555.3058560

[10] E. Freeman, G. Wilson, S. Brewster, G. Baud-Bovy, C. Magnusson, and H. Caltenco, "Audible Beacons and Wearables in Schools: Helping Young Visually Impaired Children Play and Move
Independently," in Proceedings of the 2017 CHI Conference on Human Factors in Computing Systems, ser. CHI '17. New York, NY, USA: ACM, 2017, pp. 4146-4157. [Online]. Available: http://doi.acm.org/10.1145/3025453.3025518

[11] Y. Guo, W. Wang, and X. Chen, "FreeNavi: Landmark-Based Mapless Indoor Navigation Based on WiFi Fingerprints," in 2017 IEEE 85th Vehicular Technology Conference (VTC Spring), June 2017, pp. 1-5. [Online]. Available: https://doi.org/10.1109/VTCSpring.2017.8108350

[12] L. Zeng and G. Weber, "A pilot study of collaborative accessibility: How blind people find an entrance," in MobileHCI '15. ACM, 2015, pp 347-356. [Online]. Available: https://doi.org/10.1145/2785830.2785875

[13] J. Balata, Z. Mikovec, and P. Slavik, "Landmark-enhanced route itineraries for navigation of blind pedestrians in urban environment," Journal on Multimodal User Interfaces, Apr 2018. [Online]. Available: https://doi.org/10.1007/s12193-018-0263-5

[14] J. Vystrčil, Z. Míkovec, and P. Slavík, "Naviterier - indoor navigation system for visually impaired," Smart Homes 2012, 2012.

[15] J. Balata, J. Berka, and Z. Mikovec, "Indoor-Outdoor Intermodal Sidewalk-Based Navigation Instructions for Pedestrians with Visual Impairments," in International Conference on Computers Helping People with Special Needs. Springer, 2018, pp. 292-301. [Online] Available: https://doi.org/10.1007/978-3-319-94274-2 41

[16] WHO, "ICD update and revision platform: change the definition of blindness," 2009. [Online]. Available: http://www.who.int/blindness/Change the Definition of Blindness.pdf 